\title{
Hand Sanitizer: Effectiveness \& Characterization
}

\author{
Dr. Seema Tiwari \\ Dept. of ASGE, Army Institute of Technology, \\ Pune. Dighi Hills, Alandi Road, \\ Pune. (411015).
}

\author{
Nishu Rai \\ Dept. of ASGE, Army Institute of Technology, \\ Pune. Dighi Hills, Alandi Road, \\ Pune.(411015).
}

\author{
C. Kushi \\ Dept. of ASGE,Army Institute of Technology, \\ Pune. Dighi Hills, Alandi Road, \\ Pune.(411015).
}

\begin{abstract}
Alcohol based sanitizers are often compared with the traditional method of washing hands with soap. So some of the major advantages of sanitizers over soap are Hand sanitizer can be more portable and accessible when people are on the go, which can increase the number of times they are able to disinfect their hands. This can help reduce the likelihood of transmitting viruses, it can often be the most convenient option.The benefit of hand sanitizer is the ability to combat germs when water and soap are not immediately available.
\end{abstract}

Keywords-Hand sanitizer; antimicrobial; antiseptic agents; fingerpad

\section{INTRODUCTION}

Hand sanitizer is a liquid or gel generally used to decrease infectious agents on the hands. They are available as liquids, gels, and foams. The paper focuses on addressing various pros and cons of Alcohol based hand sanitizer (ABHS). Data suggests that more than 1.4 million patients in developed and developing countries worldwide are affected at any time with healthcare-associated infections (HCAI).

According to the Center for Disease Control (CDC) hand hygiene encompasses the cleansing of your hands by using hand washing with soap and water, antiseptic hand washes, antiseptic hand rubs such as alcohol-based hand sanitizers (ABHS), foams or gels, or surgical hand antisepsis.For many reasons, alcohol hand sanitizers are increasingly being used as disinfectants over hand washing with soap and water. Their ease of availability, no need for water or plumbing, and their proven effectiveness in reducing microbial load are just a few.

According to the Centers for Disease Control and Prevention (CDC), alcohols have excellent in vitro germicidal activity against gram-positive and gram-negative vegetative bacteria, including multidrug-resistant pathogens (MRSA, VRE), Mycobacterium tuberculosis, HIV, influenza virus, $\mathrm{RSV}$, vaccinia, and hepatitis $\mathrm{B}$ and $\mathrm{C}$ viruses.

One concern is that the antimicrobial effect of ABHS is very short lived. ABHS are tremendously effective in preventing the spread of the seasonal flu, H1N1, URI, and other viral-based and bacterial-based diseases. Another advantage to using ABHS is that they are often less irritating to the hands. Hand sanitizers may not be as effective when hands are visibly dirty or greasy.
Depending on the active ingredient used, hand sanitizers can be classified as one of two types: alcohol-based or alcohol-free. Alcohol-based products typically contain between 60 and 95 percent alcohol, usually in the form of ethanol, isopropanol, or $n$-propanol. At those concentrations, alcohol immediately denatures proteins, effectively neutralizing certain types of microorganisms.

Alcohol-free products are generally based on disinfectants, such as benzalkonium chloride (BAC), or on antimicrobial agents, such as triclosan. The activity of disinfectants and antimicrobial agents is both immediate and persistent. Many hand sanitizers also contain emollients (e.g., glycerin) that soothe the skin, thickening agents, and fragrance.

\section{MATERIALS AND METHODS}

Most alcohol-based hand antiseptics contain isopropanol, ethanol , n-propanol, or a combination of 2 of these products. The antimicrobial activity of alcohols can be attributed to their ability to denature and coagulate proteins. The microorganism's cells are then lysed, and their cellular metabolism is disrupted. Alcohol solutions containing 60\% to 95\% alcohol are most effective. Notably, higher concentrations are less potent because proteins are not denatured easily in the absence of water. Alcohol concentrations in antiseptic hand rubs are often expressed as percent by volume. The highest antimicrobial efficacy can be achieved with ethanol (60\% to $85 \%)$, isopropanol (60\% to $80 \%)$, and n-propanol (60\% to $80 \%)$.

Ethanol, the most common alcohol ingredient, appears to be the most effective against viruses; whereas, the propanols have a better bactericidal activity than ethanol. None of the alcohols has shown a potential for acquired bacterial resistance. The combination of alcohols may have a synergistic effect.

The concentration of alcohol does change the efficacy with one study showing a hand rub with $85 \%$ ethanol being significantly better at reducing bacterial populations compared to concentrations of $60 \%$ to $62 \%$. ABHS also often contain humectants, like glycerin, which help prevent skin dryness, and emollients or moisturizers, like aloevera, which help replace some of the water that is stripped by the alcohol.

Efficacy is also very dependent on the technique of application of the alcohol hand sanitizer. One must apply the 
product to the palm and rub the product all over the surfaces of both hands until they are dry. There have been several studies comparing the amount needed to be effective (2.4 to 3 $\mathrm{mL}$ is recommended) and application time required to achieve hand disinfection ( 25 to 30 seconds). Another study found that high-quality hygienic hand disinfection is not possible within 15 seconds and that a 30 -second application time of ABHS is recommended.

COVID-19 pandemic resulted into spreading of awareness about hygine and the hand sanitizer and mask in medical strores ran out of stock. To overcome this we researched about the manufacturing of alcohol based hand sanitizer.

\section{RESULT AND DISCUSSION}

The FDA recommends consumers look for up to 95 percent ethanol or isopropanol .Michael Chang, an infectious disease specialist with UTHealth at the University of Texas in Houston, says there are some hand sanitizers with an ingredient called benzalkonium chloride, which has been shown to reduce bacteria and viruses, but not as much as alcohol-based sanitizers. "Interestingly, benzalkonium chloride is active against norovirus, but for most respiratory viruses, like seasonal flu or this new [COVID-19 coronavirus], the alcohol-based hand sanitizers are preferred."

Now , coming to some of the limitations of alcohol based sanitizer, hand sanitizers are effective at neutralizing many microbes, viruses, and bacteria - but not all. Hand sanitizers are active against all types of viruses except norovirus, which causes a certain type of diarrhea, Sanitizers also don't protect against some types of bacteria, including one called C. difficile, which causes diarrhea from antibiotic overuse.

Ethanol-based hand sanitizers can cause alcohol poisoning if a person swallows more than a couple of mouthfuls. US poison control centers received nearly 85,000 calls between 2011 and 2015 about hand sanitizer exposures among children. Several studies have found that the ingestion of ethanol from hand sanitizers can induce intoxication and hypoglycemia in children. Older children have been known to swallow hand sanitizers to become drunk purposefully.

\section{A. Effectiveness}

The effectiveness of hand sanitizer depends on multiple factors, including the manner in which the product is applied e.g., quantity used, duration of exposure, frequency of use and whether the specific infectious agents present on the person's hands are susceptible to the active ingredient in the product.

In general, alcohol-based hand sanitizers, if rubbed thoroughly over finger and hand surfaces for a period of 30 seconds, followed by complete air-drying, can effectively reduce populations of bacteria, fungi, and some enveloped viruses e.g., influenza A viruses similar effects have been reported for certain alcohol-free formulations, such as $\mathrm{SAB}$ hand sanitizer.
Most hand sanitizers, however, are relatively ineffective against bacterial spores, non-enveloped viruses e.g., norovirus, and encysted parasites e.g., Giardia. They also do not fully cleanse or sanitize the skin when hands are noticeably soiled prior to application.

The following in vivo methods use experimental contamination to test the capacity of a formulation to reduce the level of transient microflora on the hands without regard to the resident flora. The formulations to be tested are hand antiseptic agents intended for use by HCWs, except in the surgical area.

a) CEN standards: EN 1499 and EN 1500

In Europe, the most common methods for testing hygienic hand antiseptic agents are EN 1499 and EN 1500.Briefly, the former standard requires $12-15$ subjects, and the latter (in the forthcoming amendment) 18-22, and a culture of $E$. coli. Subjects are assigned randomly to two groups where one applies the test formulation and the other a standardized reference solution. In a consecutive run, the two groups reverse roles (cross-over design).

If an antiseptic soap has been tested according to EN 1499 , the mean $\log _{10}$ reduction by the formulation must be significantly higher than that obtained with the control (soft soap). For handrubs (EN 1500), the mean acceptable reduction with a test formulation shall not be significantly inferior to that with the reference alcohol-based handrub (isopropyl alcohol or isopropanol $60 \%$ volume).

\section{b) ASTM standards}

\section{ASTM E-1174}

Currently, handwash or handrub agents are evaluated using this method in North America. The efficacy criteria of the FDA's Tentative Final Monograph (TFM) are a 2$\log _{10}$ reduction of the indicator organism on each hand within 5 minutes after the first use, and a $3-\log _{10}$ reduction of the indicator organism on each hand within 5 minutes after the tenth use.The performance criteria in EN 1500 and in the TFM for alcohol-based handrubs are not the same. Therefore, a formulation may pass the TFM criterion, but may not meet that of EN 1500 or vice versa. It should be emphasized here that the level of reduction in microbial counts needed to produce a meaningful drop in the hand-borne spread of nosocomial pathogens remains unknown.

\section{ASTM E-1838}

The fingerpad method can be applied with equal ease to handwash or handrub agents. When testing handwash agents, it can also measure reductions in the levels of viable virus after exposure to the test formulation alone, after posttreatment water rinsing and post-rinse drying of hands. This method also presents a lower risk to subjects because it entails contamination of smaller and well-defined areas on the skin in contrast to using whole hands (see below). The method can be applied to traditional as well as more recently discovered viruses such as caliciviruses. 


\section{ASTM E-2276}

This method is for testing handwash or handrub against bacteria. It is similar in design and application to the method E-1838 described above for working with viruses.

above for working with viruses (E-1838) and bacteria (E2276).

\section{ASTM E-2011}

In this method, the entire surface of both hands is contaminated with the test virus, and the test handwash or handrub formulation is rubbed on them. The surface of both hands is eluted and the eluates assayed for viable virus.

\section{ASTM E-2613}

This method is for testing handwash or handrub against fungi. It is similar in design and application to the methods described

\section{CONCLUSION}

Alcohol based hand sanitizers are easy to prepare but the must be used after proper testing of the effectiveness. The sanitizers are effective against most of the bacteria and viruses.Using hand sanitizer is the easiest way and handy way to keep your hands hygienic anywhere and everywhere.

\section{ACKNOWLEDGEMENT}

We show our sincere thank to Director ,Joint Director , Principal and HOD ASGE of Army Institute of Technology, Dighi,Pune for their continuous motivation and providing all the facilities to make this project successful.

\section{REFERENCES}

[1] U.S. Centers for Disease Control and Prevention Vessel Sanitation Program, "OPRP - General Information on Hand Hygiene," information sheet (July 2009), accessed March 9, 2015.

[2] Amy Simonne, "Hand Hygiene and Hand Sanitizers," publication no. FCS8788 in a series from the Family Youth and Community Sciences Department, Florida Cooperative Extension Service, Institute of Food and Agricultural Sciences, University of Florida (March 2011), accessed March 9, 2015.

[3] Raymond Li, "Alcohol-Based Hand Sanitizer Safety,” B.C. Drug and Poison Information Centre (2010), accessed March 11, 2015.

[4] "Things About Hand Sanitizers," Berkeley Wellness, University of California, Berkeley (September 8, 2014), accessed March 17, 2015. 